\title{
Crosstalk reduction in fiber links using double polarization
}

\author{
Nada Badraoui ${ }^{1} \cdot$ Tibor Berceli $^{1}$ \\ Received: 9 October 2019 / Accepted: 21 February 2020 / Published online: 24 March 2020 \\ (c) The Author(s) 2020
}

\begin{abstract}
The fiber optic link is one of the main segments of up-to-date telecommunication systems. Developing low cost, ultra reliable systems with very low latency for massive machine to machine and enhanced broadband mobile networks is a hot research topic. Motivated by this we investigated the polarization multiplexing technique applicable in fiber optic links because it can be used for increasing the transmission capacity. In the present paper a novel approach, the double polarization multiplex method is investigated. In that case the crosstalk between two polarization states is one of the main issues. As a result of crosstalk, the two channels composed by double polarization can disturb each other significantly. Crosstalk can appear due to any imperfection in the fiber and in the optical circuitry of the transmitter and receiver. In this paper, we propose a new method for reducing the effect of crosstalk even in the case of high-speed long-distance transmission. The applicability of the new concept is validated by experiments. A bit error rate better than $10^{-6}$ has been achieved over a $7 \mathrm{~km}$ long fiber carrying $12 \mathrm{Gbit} / \mathrm{s}$ NRZ modulation. That result is significantly better than the already published experimental data using 2, $5 \mathrm{Gbit} / \mathrm{s}$ bit rate. The cross polarization discrimination is also improved by $10-15 \mathrm{~dB}$.
\end{abstract}

Keywords Crosstalk reduction $\cdot$ Double polarization multiplex $\cdot$ Coherent optical link

\section{Introduction}

The fiber optic link is one of the basic segments of up-to-date telecommunication systems. Developing low cost, ultra reliable optical systems with very low latency for massive machine to machine and enhanced broadband mobile connections is a hot research topic. Motivated by that research goal we apply double polarization multiplexing technique in fiber optic links for increasing its capacity. The applicability of the new concept is validated by experiments.

The deployment of an optical cable containing several fibers is a very expensive investment. Therefore, increasing the capacity of existing optical links is an important issue

Tibor Berceli

berceli@hvt.bme.hu

Nada Badraoui

nada@hvt.bme.hu

1 Budapest University of Technology and Economics, Budapest, Hungary 
(Al-Raweshidy and Shozo 2002). For that purpose, polarization division multiplex (PDM) is an effective approach (Popovskyy and Iskandar 2016; Ivanovich et al. 2018). Polarization division multiplexing is applied together with wavelength divison multiplexing (WDM) to decrease the separation of adjacent channels. The polarization of the adjacent channels is orthogonal which means periodicity in the state of polarization. This way the wavelength difference between the adjacent channels can be reduced resulting in capacity enhancement.

The polarization division multiplexing technique has already been investigated in some publications (Ivanovich et al. 2018; Nelson et al. 2001). Most of them were theoretical studies or simulations (Core 2006; Goossens et al. 2017; Badraoui and Berceli 2019b). Many papers discussed the polarization problems in fibers (Kaminow 1981; Hsiao et al. 2012; Zayats 2018). However, the double polarization approach has not been discussed in details yet. First of all, an important issue, cross talk between orthogonally polarized waves has not been investigated using experimental connections.

In the present paper a novel approach, the double polarization multiplex (DPM) is investigated. In that case the optical beam has simultaneously two orthogonal polarizations or by other words it has double polarization. In the orthogonal polarizations of the beam the information to be transmitted is different. We present experimental procedures to validate some challenging issues. One of them is the crosstalk. For reducing crosstalk a new approach is suggested. By applying that method in the experiments the achieved measurement results proved the applicability of the new approach in links for high-speed longdistance transmission.

\section{Principle of investigation}

The deployment of an optical cable containing several fibers is a very expensive investment. Therefore, incraesing the capacity of existing optical links is an important issue. For that purpose, the double polarization multiplexing (DPM) is a cost-effective approach. In the DPM approach the optical beams have simultaneously two orthogonal polarizations providing this way two independent high speed channels.

There are two different methods for generating polarization multiplexing. In the incoherent system, there are two independent lasers with significantly different wavelengths. Therefore, by sufficient optical filtering, the effect of crosstalk can be substantially reduced or almost eliminated.

That procedure cannot be applied in the case when we have only a single laser source which means a coherent system. In this case the two beams with perpendicular polarization are produced from the beam of a single laser using a polarization beam splitter. In this system optical filtering cannot be applied for reducing the effect of crosstalk.

The crosstalk between the two channels with orthogonal polarizations is a difficult problem. As a result of crosstalk, the two channels can disturb each other significantly. Crosstalk can appear due to any imperfection in the fiber and in the optical circuitry of the transmitter and receiver. To reduce the effect of crosstalk the generation of the individual waves should be perfect which means each of them have to have only a single polarization. In spite of these precautions during propagation along the fiber the polarization mode dispersion causes crosstalk impairing signal transmission.

In this paper, we present a new approach for reducing the effect of crosstalk even in the case of high-speed long-distance transmission. As a counter-measure to the crosstalk 


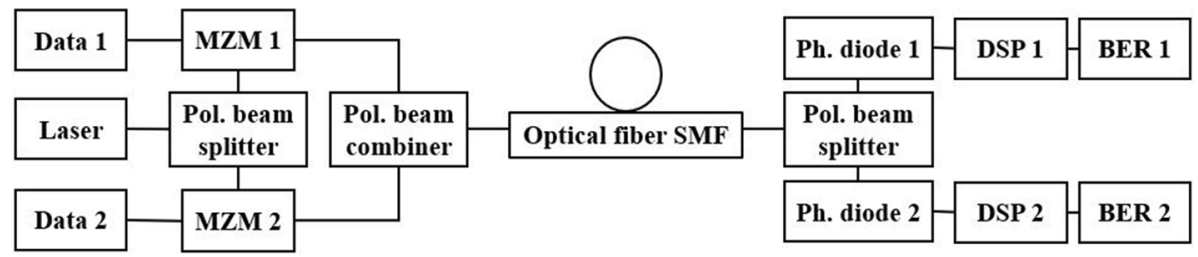

Fig. 1 Basic block diagram of an optical fiber link using double polarization multiplexing

problem we introduce $90^{\circ}$ phase shift between the optical beams in the two channels. That way we have two coherent orthogonal beams in the time or frequency domain. If that orthogonality is kept along the fiber then the effect of crosstalk is significantly reduced. During our experimental investigations we utilized the orthogonality of the optical waves with success.

The new concept has been investigated by an experimental set-up. The coherent optical polarization multiplexing link is presented by the basic block diagram of Fig. 1. The beam of a single laser diode is transfered into a beam with perfect single polarization applying a polarization controller. That wave is led to a polarization beam splitter which produces two beams each of them with single polarization and their polarizations are orthogonal. These optical waves are modulated by different information in Mach-Zehnder modulators (MZM). Then the modulated beams are combined by a polarization beam combiner and put on a single mode fiber. At the reception side, the two channels with orthogonal polarization are separated by a polarization beam splitter, detected and processed. This way we can measure the quality of signal transmission. The parameters of the experimental set-up are listed in Table 1 in the Appendix.

\section{Properties of the components}

Polarization is a property of electromagnetic waves which describes the orientation of their oscillations at a given point in space. Understanding the physics of certain optical components (fibers, polarizers, polarization controllers, beam splitter, beam combiner, etc.) requires a good knowledge of the basic concepts related to polarization.

\subsection{Polarization controller}

Starting with the experimental study, we investigated the behaviour of polarisation controller (PC) type PC-HP 8169A which is needed for polarization sensitive devices like Mach-Zehnder modulator, laser sources, etc. Moreover, the polarization controller is a perfect device to create all the possible states of polarization with easy and speedy manner. The laser beam has elliptical polarization. Therefore its beam is passed through a linear polarizer plate in the polarization controller to create perfect linear polarization wave from the laser beam. Then by the quater wave $(\lambda / 4)$ and half wave $(\lambda / 2)$ plates the state of polarization (SOP) is adjusted.

The half wave $(\lambda / 2)$ plate with its axis oriented at $0^{\circ}$ will rotate the linear polarization to horizontal polarization and the quater wave $(\lambda / 4)$ plate oriented at $90^{\circ}$ will rotate the linear polarization to vertical polarization. The wavelength $\lambda=\alpha \sin \omega / 2(\alpha$ is the orientation of 
the axes, $\omega$ is the rotation for the angle) is illustrated in Fig. 2. That figure shows how the PC-HP 8169A type polarization controller creates the relative states of polarization points according to a specified path. Figure 2 also shows the orthogonal great circles on the Poincaré sphere at $\lambda / 2$ and $\lambda / 4$. PC-HP 8169 A ensures high polarization extinction ratio (PER) for both orthogonal polarizations.

The polarization controller (PC) connected to the laser output is used to rotate the polarization angle of laser beam. This angle influences the operation of the polarization beam splitter (PBS) which is connected to the output of polarization controller. The rotation angle was varied to get maximum power for each channel as it is shown in Fig. 3. The maximum power of $2.56 \mathrm{dBm}$ has been achieved with $0^{\circ}$ phase of half-wave plate and $90^{\circ}$ phase of quater-wave plate. The power loss of the polarization controller was around $2.5 \mathrm{~dB}$.

\subsection{Polarization beam splitter}

The polarization beam splitter (PBS) has many advantages besides its flexible design: it has a high power handling capability with high polarization extinction ratio and it is highly modular. It contains two inputs ( $\mathrm{R}$ and $\mathrm{T}$ ) and two outputs (1 and 2). Figure 4 presents the photo (A) and the standard orientations of the fibers (B) in the polarization beam splitter or combiner. As a passive device it can be used either for combining or splitting the beams.

We measured the signal power at the outputs 1 and 2 of the PBS. The goal in this step was to find the maximum and the minimum output power as well as the adjustment when the two outputs are equal and of high power. These adjustments are dependent on three parameters: the linear polarizer which is used to adjust the linear polarization phase, and on the quater wave $(\lambda / 4)$ plate and the half wave $(\lambda / 2)$ plate. Figure 5 illustrates the relation between the output power and the polarization phases.

The measurements were performed in different ranges of phase shift $\left(0^{\circ}-105^{\circ}\right)$. We noticed that the best isolation between the two channels was obtained when the linear polarization filter phase was $50^{\circ}$ and the polarization phase was $103^{\circ}$. The maximum and

Fig. 2 Poincaré sphere

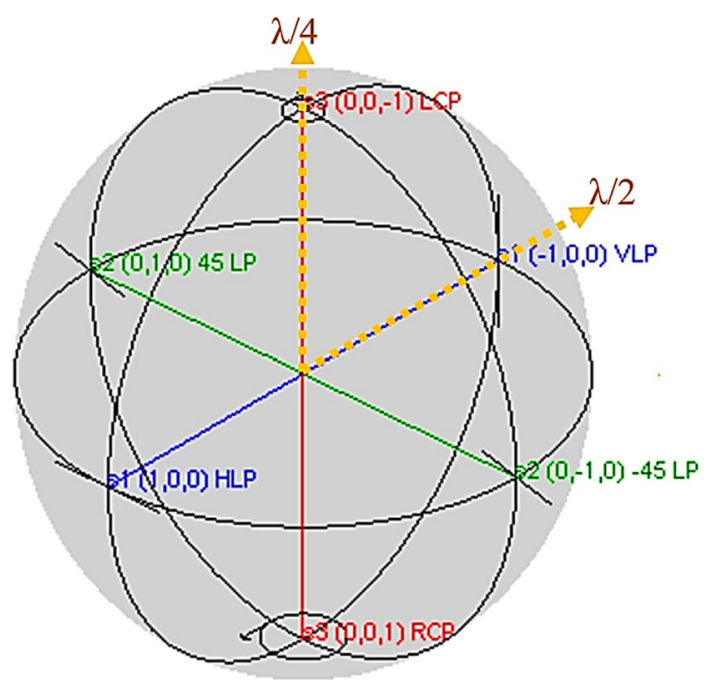


Fig. 3 Output power of the channels versus the phase shift of linear plate of polarization controller

Fig. 4 A: photo and B: standard orientation of polarization maintaining fibers in polarizing beam splitter or combiner

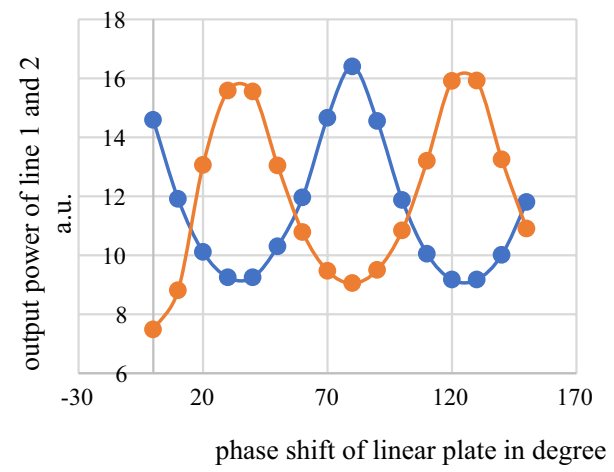

A

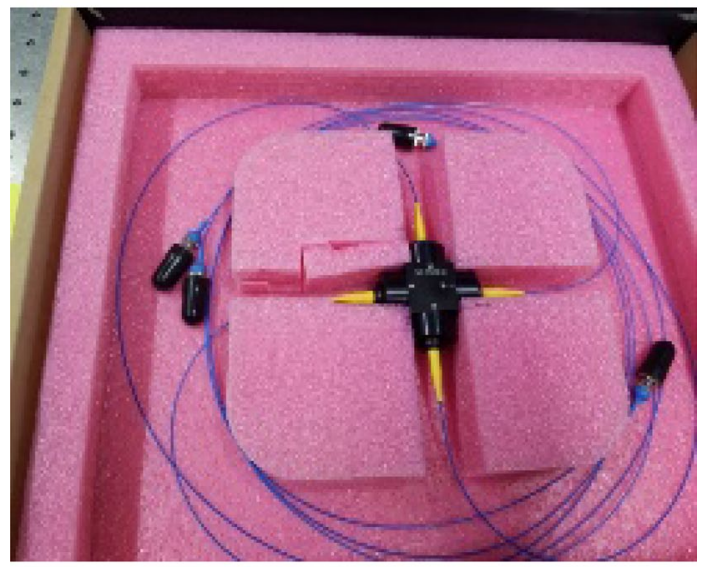

B

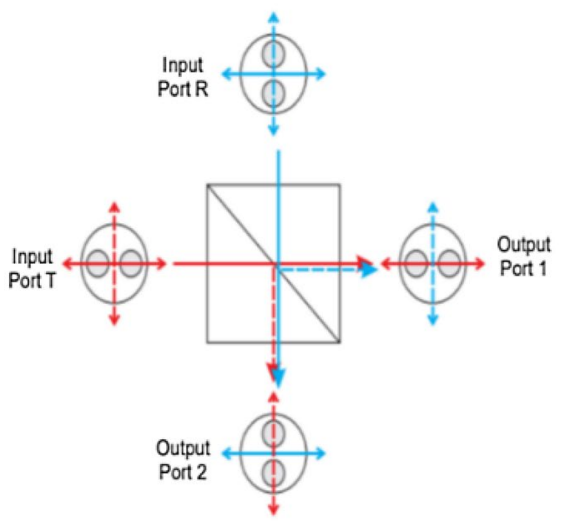

minimum power values are $-0.6 \mathrm{dBm}$ and $-34.36 \mathrm{dBm}$ for the 1 st output and for the $2 \mathrm{nd}$ output, repectively. The isolation was $33.76 \mathrm{~dB}$ providing proper separation between the channels. 


\subsection{Polarization mode dispersion}

One important task is to manage the polarization mode dispersion effect. When we have a wave with polarization that polarization is accompanied by a perpendicular polarization. The phase velocities of these two waves are different which results in impaired signal at reception. That effect is called polarization mode dispersion (PMD). That is dependent on the ratio of the intensities of two orthogonal polarized waves which is called polarization extinction ratio (PER). The effect of PMD has been investigated by simulation (Badraoui and Berceli 2019a, b). Good performance has been achieved if PER was kept above $22 \mathrm{~dB}$. In a polarization multiplexing approach each polarization has to meet that requirement.

The high polarization extension ratio is necessary to reduce the effect of polarization mode dispersion. When PER is not high enough then beside the useful polarization the optical wave has a spurious perpendicular polarization with some intensity. The phase velocities of the two polarization components are different, i.e. PMD causes pulse broadening. Due to pulse broadening we get inter-symbol interference (ISI) which deteriorates the bit error rate (BER).

We investigated the effect of PER on the bit error rate by simulation (Badraoui and Berceli 2019b). The simulation results show the PER and the polarization crosstalk are inversely proportional to each other (Badraoui and Berceli 2019b). That means as the polarization extinction ratio increases the bit error rate improves resulting in better performance. In our experiment, the PER was kept around $25 \mathrm{~dB}$.

\subsection{Mach-Zehnder modulator}

Two Mach-Zehnder modulators (MZM) were used with ON-OFF keying modulation. The modulation amplitudes were $2 \mathrm{~V}_{\mathrm{p} \text {-p }}$ at the quadratic point of the two MZMs which were

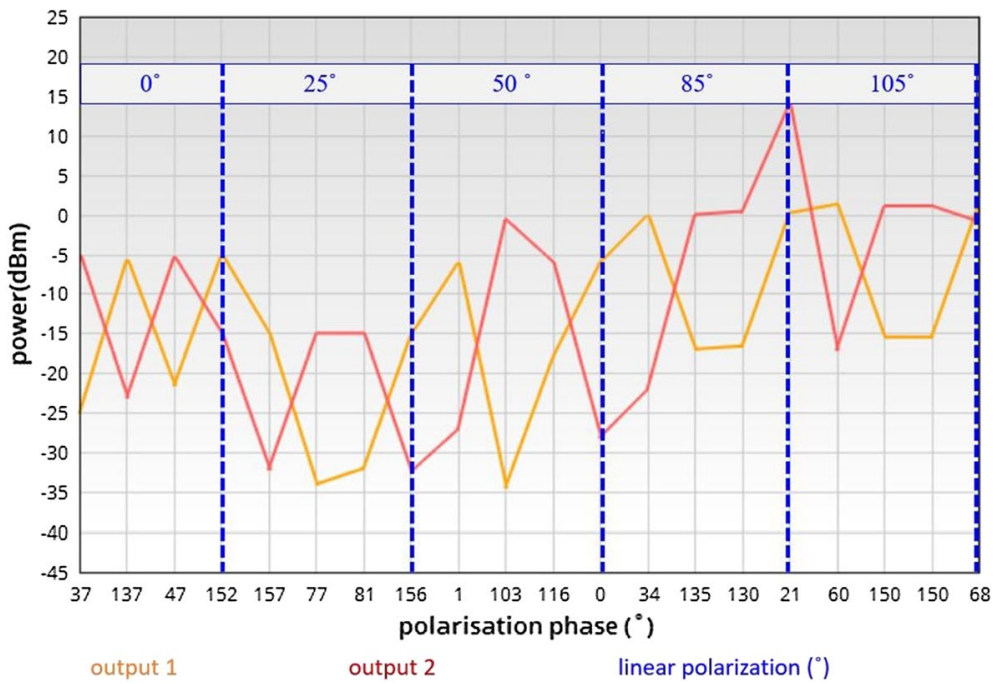

Fig. 5 Power output of the channels: output 1: red curve, output 2: yellow curve. As a parameter the blue numbers show the phase of linear plate in degree 
$-1.8 \mathrm{~V}$ and $-1.6 \mathrm{~V}$ for the 1 st and 2nd MZM, respectively. We applied two polarization controllers (PC) before and after the MZMs because they are very sensitive to the polarization state. The two outputs of the MZMs were combined with a polarization beam combiner (PBC) as shown in Fig. 6.

\section{Experimental link}

The block diagram of the double polarization multiplexing experiment is shown in Fig. 6. In the experiment between the transmitter and receiver the signal propagates through a single mode (SMF) optical fiber. An optical amplifier is also applied in the receiver because the transmitter power is decreased by $11 \mathrm{~dB}$. In the receiver we have a polarization beam splitter to separate the two beams with different polarizations to test the BER and the eye diagram of the two channels.

\subsection{Bit error rate dependence on optical wavelength}

The diagram in Fig. 7 shows the relationship between the BER and the laser wavelength. The wavelength ranges from 1400 to $1580 \mathrm{~nm}$ ) while the power equals $7 \mathrm{dBm}$ taken into consideration the amplification power. Although the attenuation coefficient of the optical fibers is low $(0.2 \mathrm{~dB} / \mathrm{km}$ in the telecommunication band at $1550 \mathrm{~nm})$, long-distance links require the use of amplifiers. We recognize from the diagram in Fig. 7 that the best range to have a good transmission with BER equal to $10^{-11}$ is in the wavelength range of $1545-1555 \mathrm{~nm}$. The measurement error is around $10^{-11}$.

\subsection{Dependence of BER on bit rate}

Figure 8 shows the dependence of BER on bit rate. The bit rate range starts from $0.1 \mathrm{Gbit} / \mathrm{s}$ and extends to $12 \mathrm{Gbit} / \mathrm{s}$; the wavelength is $1550 \mathrm{~nm}$ and the optical power is $7 \mathrm{dBm}$. The fiber length is $7 \mathrm{~km}$. The bit error rate (BER) is used to evaluate the quality of optical transmission. It can be determined by comparing the transmitted and received binary data sequences as it is defined by the ratio of the number of error bits to the total number of bits transmitted.

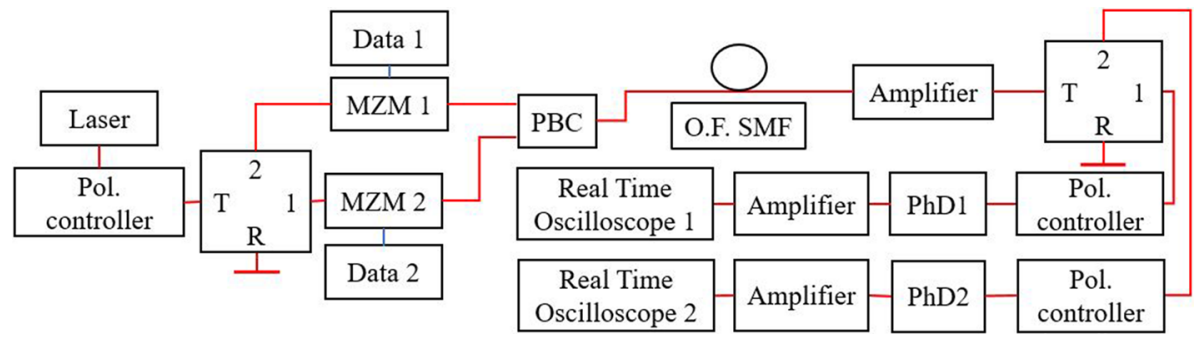

Fig. 6 Block diagram of the polarization multiplexing experiment 
Fig. 7 Impact of optical wavelength on bit error rate

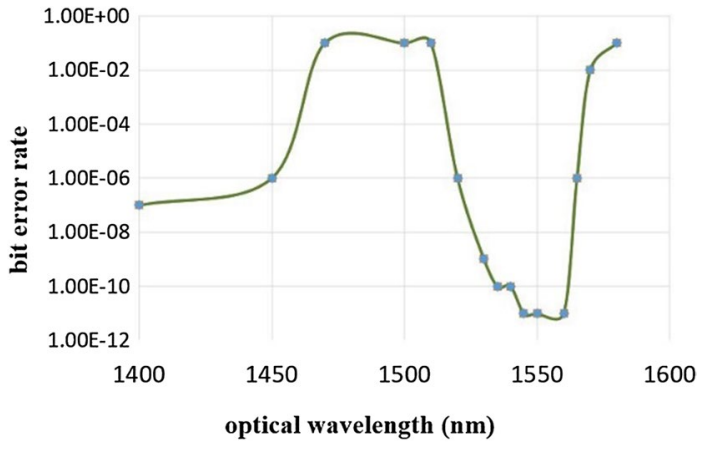

\subsection{Measurement of bit error rate as a function of the received power}

We study the bit error rate as a function of optical power reaching the photo detectors in the receiver. Measuring an optical fiber with length of $25 \mathrm{~km}$, bit rate of $12 \mathrm{Gbit} / \mathrm{s}$ we received bit error rate better than $10^{-10}$ when the amplification was in the range of linear operation. We get inverse relation between optical amplification and BER. As more we increase the amplification beyond a limit the more the signal becomes distorted due to nonlinearity. When the optical power exceeds the average value, the pulses undergo a small contraction. However, if the average power is too low, the non-linear effects can be neglected compared to the effect of PMD.

\subsection{Measurement of bit error rate as a function of fiber length}

The fiber length influences the transmission quality significantly. In the double polarization multiplex application usually the length of fiber is not too long. Results of measurements are presented in Fig. 9. Bit error rate is depending on two parameters: fiber length and bit rate. The fiber length was: $0 \mathrm{~km}, 1 \mathrm{~km}, 2 \mathrm{~km}, 7 \mathrm{~km}$ and $25 \mathrm{~km}$. The bit rate was: $1 \mathrm{Gbit} / \mathrm{s}$, $2 \mathrm{Gbit} / \mathrm{s}, 4 \mathrm{Gbit} / \mathrm{s}, 8 \mathrm{Gbit} / \mathrm{s}, 12 \mathrm{Gbit} / \mathrm{s}$. For each case, we adjusted the clock generator time delay and the threshold margin of data detection to improve the bit error rate and the eye diagram. As an example Fig. 10 shows the eye diagram in case when the fiber length is $7 \mathrm{~km}$ and the bit error rate is $10^{-10}$.

Fig. 8 Bit error rate as function of bit rate

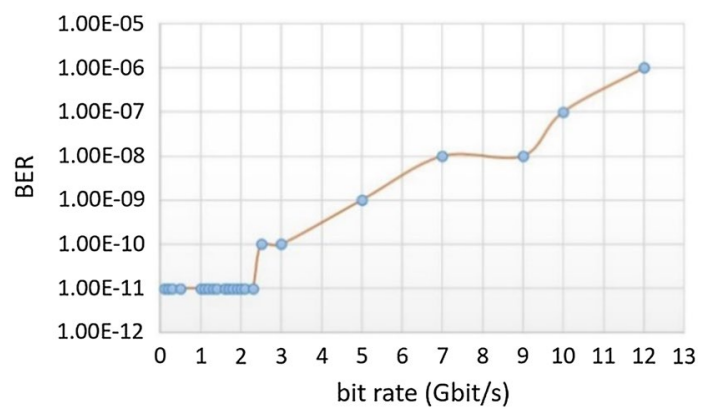


Fig. 9 Impact of fiber length and bit rate on bit error rate
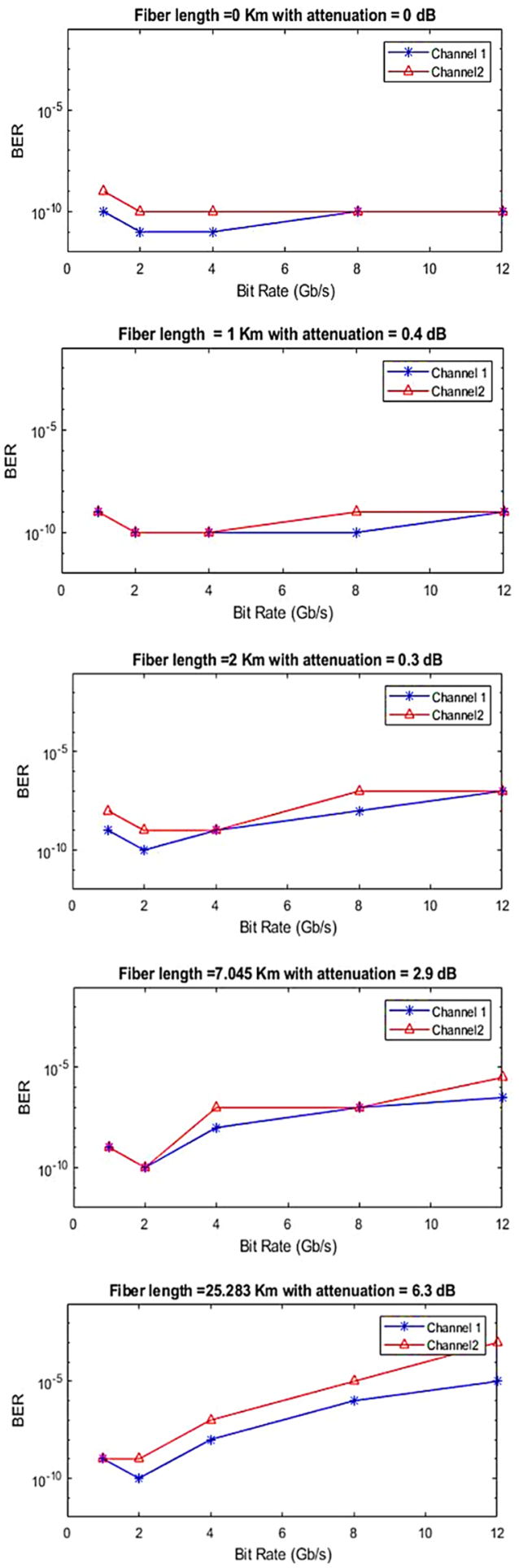
Fig. 10 Eye diagram when the fiber length is $7 \mathrm{~km}$ and bit error rate is $10^{-10}$

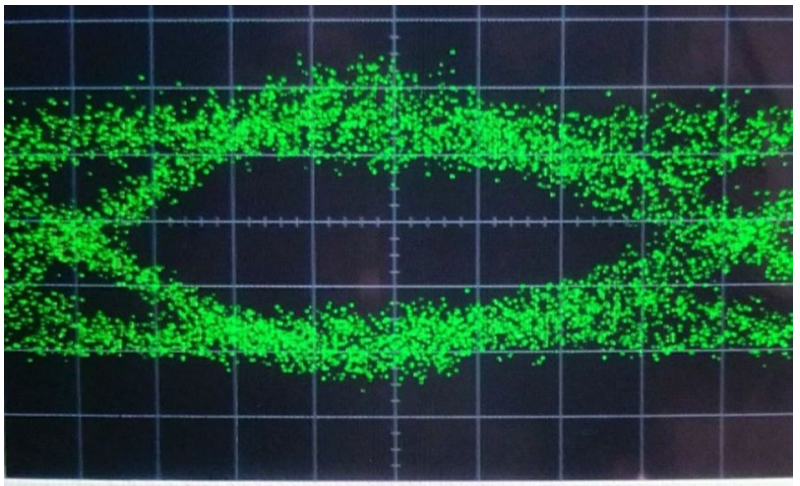

\subsection{Evaluation of measured data}

The data of Fig. 9 were measured in two steps. In the first step channel 1 was active and channel 2 was inactive. That means we had only one polarization. The results are presented by blue lines. For characterizing the results we used the bit error rate as very sensitive data. Below $2 \mathrm{~km}$ distance the bit error rate is not significantly dependent on fiber length and on bit rate. However, at longer distances it is noticed that the bit error rate has been slowly impaired due to attenuation and polarization mode dispersion in the fiber, as seen at $25 \mathrm{~km}$ fiber length.

In the next step of measurement both channels were active and their polarizations were orthogonal. This case is shown by red lines. At shorter distances, less than $2 \mathrm{~km}$, there is no relevant difference between the single and double polarization cases. However, at longer distances due to cross polarization the bit error rate has been more impaired. That can be seen very clearly at $25 \mathrm{~km}$ fiber length.

The effect of cross polarization is characterized by the cross polarization discrimination which can be derived from bit error rate difference between the single and double polarization cases. Evaluating the measured results no noticeable cross polarization discrimination is obtained at distances less than $2 \mathrm{~km}$. The cross polarization discrimination is about $45 \mathrm{~dB}$ for a distance of $7 \mathrm{~km}$ and $40 \mathrm{~dB}$ for a distance of $25 \mathrm{~km}$. These figures are $10-15 \mathrm{~dB}$ better than the already published $30 \mathrm{~dB}$ cross polarization discrimination (Morant et al. 2014).

\section{Conclusions}

The capacity enhancement of optical links is an important issue. For that purpose, polarization multiplexing is a cost-effective approach. In that approach the crosstalk is a substantial problem which has to be reduced significantly. In our approach two waves are created simultaneously with equal intensity and with a perfect single polarization. Then they are independently modulated and combined on a single mode fiber with precisely orthogonal polarization. In spite of these precautions during propagation along the fiber polarization mode dispersion causes cross- talk impairing signal transmission.

As a countermeasure to that problem, a new approach has been applied. We introduced $90^{\circ}$ phase shift between the two optical waves. That way there are two orthogonal coherent beams in the time or frequency domain. By keeping this orthogonality the effect of 
crosstalk has been reduced significantly. A bit error rate better than $10^{-6}$ has been achieved using a $7 \mathrm{~km}$ long fiber with $12 \mathrm{Gbit} / \mathrm{s}$ NRZ digital modulation.

The applicability of the new concept has been validated by experiments. A bit error rate better than $10^{-6}$ has been achieved over a $7 \mathrm{~km}$ long fiber carrying $12 \mathrm{Gbit} / \mathrm{s}$ NRZ modulation. That result is significantly better than the already published experimental data using $2,5 \mathrm{Gbit} / \mathrm{s}$ bit rate. The cross polarization discrimination is also improved by $10-15 \mathrm{~dB}$ compared to the published results.

The achieved measurement results prove the applicability of the double polarization multiplex technique even in links for high-speed long-distance transmission.

Acknowledgements Open access funding provided by Budapest University of Technology and Economics (BME). The authors acknowledge Andreas Stöhr and Matthias Steeg at University of Duisburg, for the excellent consulatations and the COST project CA16220 EUIMWP for helping their research.

Open Access This article is licensed under a Creative Commons Attribution 4.0 International License, which permits use, sharing, adaptation, distribution and reproduction in any medium or format, as long as you give appropriate credit to the original author(s) and the source, provide a link to the Creative Commons licence, and indicate if changes were made. The images or other third party material in this article are included in the article's Creative Commons licence, unless indicated otherwise in a credit line to the material. If material is not included in the article's Creative Commons licence and your intended use is not permitted by statutory regulation or exceeds the permitted use, you will need to obtain permission directly from the copyright holder. To view a copy of this licence, visit http://creativecommons.org/licenses/by/4.0/.

\section{Appendix}

See Table 1.

Table 1 Parameters of experimental link

\begin{tabular}{ll}
\hline & Parameters \\
\hline SMF fiber length & $0-25 \mathrm{~km}$ \\
Bit rate & $1-12 \mathrm{Gbit} / \mathrm{s}$ \\
Optical modulation & On-off keying \\
Laser output power & $13 \mathrm{dBm}$ \\
MZM 1 quadrature point & $-1.8 \mathrm{~V}$ \\
MZM 2 quadrature point & $-1.6 \mathrm{~V}$ \\
Photodetector current & $0.45 \mathrm{~mA}$ \\
Modulation amplitude & $1-2 \mathrm{~V}_{\mathrm{p}-\mathrm{p}}$ \\
Optical wavelength & $1350-1600 \mathrm{~nm}$ \\
\hline
\end{tabular}

\section{References}

Al-Raweshidy, H., Shozo, K.: Radio over fiber technologies for mobile communications networks. Artech House (2002)

Badraoui, N., Berceli, T.: Enhancing capacity of optical links using polarization multiplexing. Opt. Quantum Electron. 51, 310-315 (2019). https://doi.org/10.1007/s11082-019-2017-3

Badraoui, N., Berceli, T.: Improvements in radio over fiber links using polarization multiplexing. In: 21th International Conference on Transparent Optical Networks (ICTON), pp. 1-6. IEEE, Angers. France (2019b) 
Core, M.T.: Cross polarization interference cancellation for fiber optic systems. IEEE J. Lightwave Technol. 24(1), 305-312 (2006)

Goossens, J.W., Yousefi, M.I., Jaouën, Y., Hafermann, H.: Polarization-division multiplexing based on the nonlinear Fourier transform. Opt. Express 25(22), 26437-26452 (2017)

Hsiao, V.K.S., Fu, W.H., Huang, C.Y., Chen, Z., Li, S., Yu, J., Zhang, J., Tang, J.: Optically switchable allfiber optic polarization rotator. Opt. Commun. 285(6), 1155-1158 (2012)

Ivanovich, D., Powell SB, Gruev, V.: Chamberlain RD. "Polarization division multiplexing for optical data communications.In: Optical Interconnects XVIII, International Society for Optics and Photonics, vol. 10538, p. 105381D. Bucharest, Romania (2018)

Kaminow, I.: Polarization in optical fibers. IEEE J. Quantum Electron. 17(1), 15-22 (1981)

Morant, M., Joaquin, P., Roberto, L.: Polarization division multiplexing of OFDM radio-over-fiber signals in passive optical networks. Adv. Opt. Technol. (2014). https://doi.org/10.1155/2014/269524

Nelson, L.E., Nielsen, T.N., Kogelnik, H.: Observation of PMD-induced coherent crosstalk in polarizationmultiplexed transmission. IEEE Photon. Technol. Lett. 13(7), 378-390 (2001)

Popovskyy, V., Iskandar, A.W.S.A.: Polarization multiplexing modulation in fiber-optic communication lines. In: 2016 Third International Scientific-Practical Conference Problems of Infocommunications Science and Technology (PIC S\&T), pp. 214-216, IEEE (2016)

Zayats, A.V.: Ultrafast polarization control with metamaterials. In: 12th International Congress on Artificial Materials for Novel Wave Phenomena (Metamaterials), pp. 463-464. IEEE (2018)

Publisher's Note Springer Nature remains neutral with regard to jurisdictional claims in published maps and institutional affiliations. 